Sir,

\section{Sildenafil (Viagra) a cause of proliferative diabetic retinopathy?}

Sildenafil is the first licensed oral drug treatment for erectile dysfunction. Sildenafil is a potent vasoactive drug whose mode of action is selective inhibition of the isoenzyme phosphodiesterase 5 (PDE5). ${ }^{1}$ Inhibition of PDE5 results in increased levels of cyclic guanosine monophosphate (cGMP), the intracellular messenger which effects vasodilation by relaxation of smooth muscle in arterioles.

Production of cGMP from guanosine triphosphate (GTP) is mediated by the nitric oxide (NO) pathway. By inhibiting PDE5, sildenafil potentiates the effects of $\mathrm{NO}$ and increases levels of cGMP (Fig. 1). ${ }^{2}$

Diabetes is a common cause of erectile dysfunction and therefore a number of our diabetic patients will inevitably be taking sildenafil. We present a case in which a long-standing insulin-dependent diabetic patient's stable background diabetic retinopathy progressed to a proliferative state within 6 months of commencing sildenafil for erectile dysfunction.

\section{Case report}

A 59-year-old was referred to the ophthalmic clinic with a 5-week history of floaters and decreased vision in his right eye. He was a patient with a history of insulindependent diabetes for 37 years, but no documented evidence of diabetic retinopathy until 1996. Since then only mild background diabetic retinopathy was noted. There was a history of a right sixth nerve palsy in 1998, which resolved spontaneously without sequelae. At his last clinic visit, 5 months prior to presentation, his vision was $6 / 6$ in both eyes. His diabetic control was deemed to be good. He maintained an average blood glucose of $7 \mathrm{mmol} / \mathrm{l}$, and his $\mathrm{HbAlc}$ was between $7 \%$ and $9 \%$ over the last 3 years. There was no evidence of renal impairment. His general health was good and he was not on any concomitant medication.

\section{Smooth muscle relaxation}

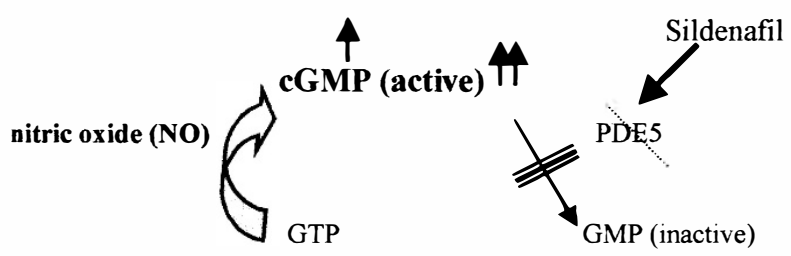

Fig. 1. Mode of action of sildenafil. Inhibition of PDE5 results in increased levels of GMP inducing further smooth muscle relaxation, and hence vasodilatation.

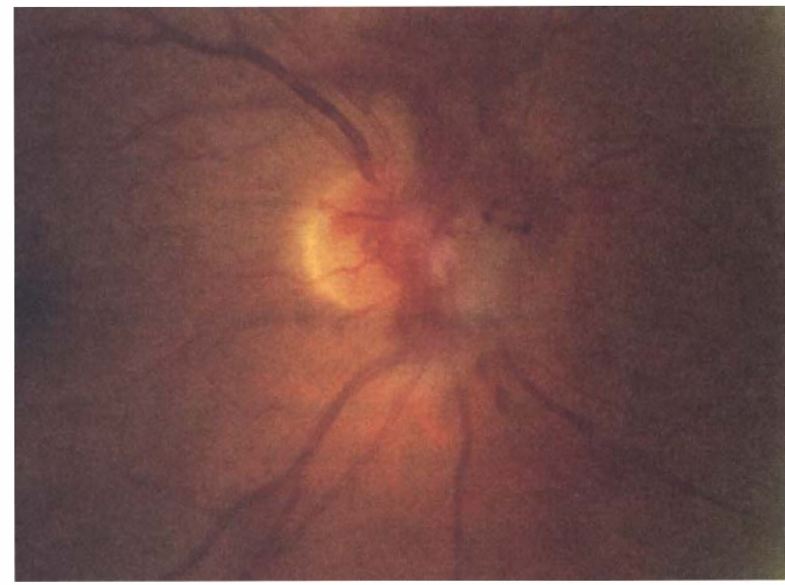

Fig. 2. Right eye, showing mild vitreous haemorrhage with a large neovascular frond emanating from the optic disc.

On examination, his visual acuity was $6 / 18$ in the right eye and $6 / 5$ in the left. There was a mild vitreous haemorrhage in the right eye with a large neovascular frond emanating from the optic disc (Fig. 2). The left eye revealed flat neovascularisation at the optic disc (Fig. 3).

In view of the unusually rapid deterioration of his diabetic retinopathy, potential causes were sought. Full cardiovascular examination and carotid Doppler scans were normal. Haematological investigations including full-blood count, erythrocyte sedimentation rate, urea and electrolytes and lipid profile were all normal.

It was not until the patient attended for laser treatment that he volunteered information regarding the use of sildenafil $100 \mathrm{mg}$ weekly. This was commenced shortly after his last routine ophthalmic appointment 5 months previously.

\section{Comment}

It is well recognised that $3-11 \%$ of patients taking sildenafil experience ocular side-effects in the form of a transient blue/green tinge to their vision, increased light

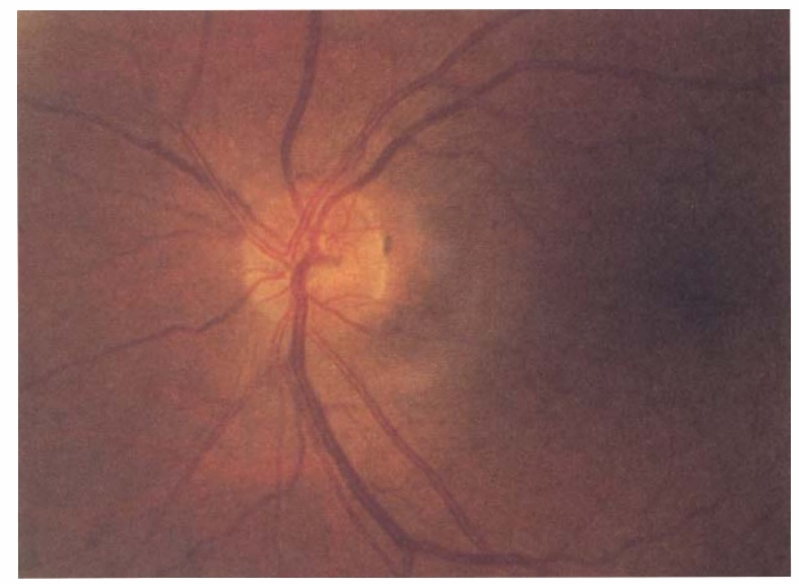

Fig. 3. Left eye, showing flat neovascularisation at the optic disc. 


\section{ANGIOGENESIS}

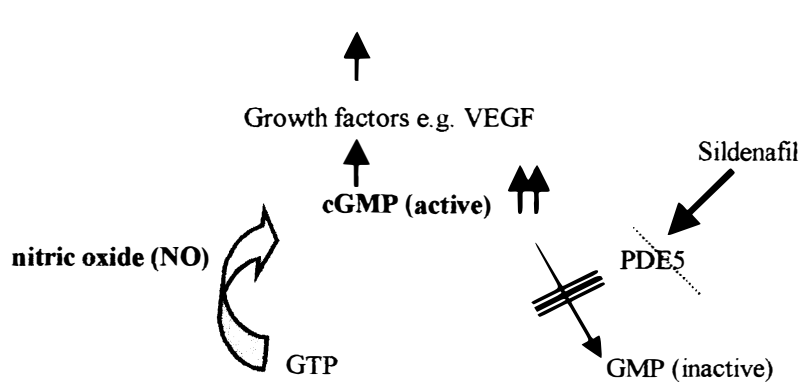

Fig. 4. Proposed mechanism for retinal angiogenesis by accumulation of GMP.

sensitivity and blurred vision. ${ }^{3}$ This is attributed to inhibition of PDE6, which is involved with the phototransduction process in photoreceptor outer segments. Electrophysiological studies have demonstrated significant decreases in a- and b-wave amplitudes $1 \mathrm{~h}$ following a $100 \mathrm{mg}$ dose of Sildenafil in healthy subjects. ${ }^{4}$ The effect resolved $6 \mathrm{~h}$ later on repeat testing.

Cardiovascular effects of sildenafil have been investigated extensively following reports of a number of cardiovascular-related deaths in the United States. ${ }^{5}$ A systematic review evaluated the safety profile of sildenafil following its use in over 4000 patients for over 6 months. ${ }^{6}$ However, there have been no published studies regarding the effects of sildenafil on animal or human subjects concerning diabetic retinopathy. Both the manufacturer (Pfizer) and the Drug Information Service were contacted and they confirm that there have been no reports of any similar cases to date.

In addition to its effects on smooth muscle relaxation, the NO/cGMP pathway is directly implicated in angiogenesis. Vascular endothelial growth factor (VEGF), a growth factor implicated in retinal neovascularisation, evokes a proliferative response on cultured microvascular endothelium of coronary post-capillary venules in vitro. ${ }^{7}$ This endothelial cell proliferative effect is reduced by pretreatment with $\mathrm{NO}$ synthase inhibitors, which reduce NO production. We suggest that the accumulation of cGMP, due to the inhibition of PDE5, may also exert a similar proliferative effect on the retinal post-capillary venules, stimulating the neovascularisation seen in proliferative diabetic retinopathy (Fig. 4).

One could argue that since the patient was an insulindependent diabetic for many years, having developed significant vascular complications in the form of a sixth nerve palsy and impotence, the development of proliferative diabetic retinopathy may simply be a reflection of the natural progression of his underlying disease. On the other hand, sildenafil is a potent vasoactive drug and diabetes fundamentally a vascular disease. Is it purely coincidence that such a dramatic deterioration in diabetic retinopathy should occur in the few months following commencement of sildenafil?
We suggest keeping an open mind regarding sildenafil and proliferative diabetic retinopathy, and stress the need for a detailed and accurate drug history in all cases.

\section{References}

1. Sildenafil for erectile dysfunction. Drug Ther Bull 1998;36(11).

2. Marmor MR. Sildenafil (Viagra) and ophthalmology [editorial]. Arch Ophthalmol 1999;117:518-9.

3. Sildenafil. Summary of drug characteristics, UK and Ireland. Pfizer Limited, September 1998.

4. Vobig MA, Klotz T, Staak M, Bartz-Schmidt KU, Engelmann U, Walter P. Retinal side-effects of sildenafil. Lancet 1999:353:375.

5. Kloner RA, Zusman RM. Cardiovascular effects of sildenafil citrate and recommendations for its use. Am J Cardiol 1999;84:N11-7.

6. Morales A, Gingell C, Collins M, Wicker PA, Osterloh IH. Clinical safety of oral sildenafil (Viagra) in the treatment of erectile dysfunction. Int J Impot Res 1998;10:69-74.

7. Morbidelli L, Chang CH, Douglas JG, Granger HJ, Ledda F, Ziche M. Nitric oxide mediates mitogenic effect of VEGF on coronary venular endothelium. Am J Physiol 1996;270:H411-5.

Anthony J.M. Burton

Anita Reynolds

Damian O'Neill

Department of Ophthalmology

Leeds General Infirmary

Leeds, UK

Mr A.J.M. Burton

Department of Ophthalmology

Leeds General Infirmary

Leeds

West Yorkshire, UK

Tel: +44 (0)1132 286288

e-mail: tony.355@virginnet.co.uk

Sir,

A new method of temporary tarsorrhaphy

Temporary tarsorrhaphy has been in use for many years as a method of coping with transient exposure problems of the cornea or persistent epithelial defects. The standard temporary tarshorrhaphy was performed by passing a silk suture through the lid margins and tying it over a bolster, but this has been replaced by the use of a monofilament nylon suture with or without a silicone button. The latter has substantially reduced the inflammatory reaction in the lid, but the opening and closing of the suture is still a traumatic procedure for the patient. A variation whereby tubes are sewn to the eyelid skin and the suture passed through the lumen of the tubes was described in 1993 by Rapoza et al. ${ }^{1}$ but is still an invasive procedure demanding local anaesthesia. The use of a cyanoacrylate to glue the lashes or lid margins together, published in 1991 by Eric et al. ${ }^{2}$ is effective, but does not allow a good examination of the cornea. The injection of botulinum toxin $\mathrm{A}$ to induce a protective ptosis in corneal disease, advocated in 1988 by Kirkness et al., ${ }^{3}$ has not been widely adopted as the ptosis is unpredictable and the toxin is expensive. 